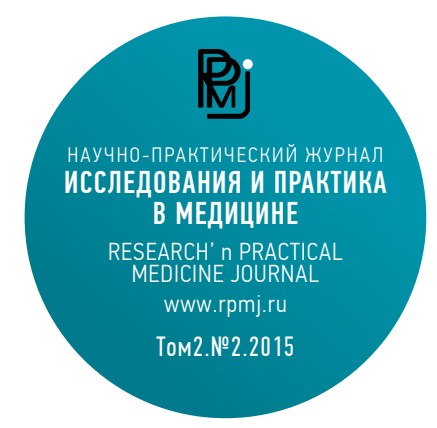

Ключевые слова:

октреотид,

аналоги соматостатина,

нейроэндокринные опухоли,

карциноидный синдром

Keywords:

octreotide,

somatostatin analogues,

neuroendocrine carcinomas,

carinoid syndrome

DOI: 10.17709/2409-2231-2015-2-2-63-72

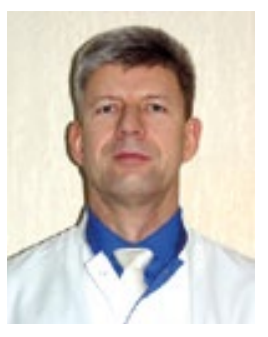

Для корреспонденции:

Моргунов Леонид Юльевич,

д.м.н., профессор кафедры терапии,

клинической фармакологии и скорой медицинской помощи ГБОУ ВПО МГМСУ имени А.И.Евдокимова Минздрава РФ

Адрес: 127473, Российская Федерация,

Москва, ул. Делегатская, д.20, стр.1

Телефон:+7 (495) 471-10-44

E-mail: morgunov.l.y@mail.ru

Статья поступила 19.04.2015,

принята к печати 11.06.2015

For correspondence:

Morgunov Leonid Yulevich,

PhD, MD, professor of therapy,

clinical pharmacology and medical emergencies

Moscow State University of Medicine and Dentistry

named after A.I. Evdokimov

Address: Ulitsa Delegatskaya, 20/1,

Moskva, 127473, Russia

Tel:+7 (495) 471-10-44

E-mail: morgunov.l.y@mail.ru

The article was received 19.04.2015

accepted for publication 11.06.2015

\section{ОКТРЕОТИД В ЛЕЧЕНИИ НЕЙРОЭНДОКРИННЫХ ОПУХОЛЕЙ: ДОСТИГНУТ ЛИ УСПЕХ?}

Моргунов Л.Ю.

ГБОУ ВПО «МГМСУ имени А.И. Евдокимова» МЗ РФ

127473, Российская Федерация, Москва, ул. Делегатская, д.20, стр.1

\section{Резюме:}

Диагностика и лечение функционирующих и нефункционирующих нейроэндокринных опухолей представляет собой сложную задачу. Единственным радикальным методом лечения этих новообразований остается хирургический, но опухоли нередко диагностируются уже на стадии метастазирования, когда возможна лишь паллиативная тактика. Октреотид у пациентов с нейроэндокринными опухолями остается препаратом с доказанной эффективностью, способным подавлять клиническую симптоматику карциноидного синдрома и обладающий антипролиферативной активностью, что позволяет улучшить качество жизни и увеличить выживаемость.

\section{OCTREOTIDE IN THE TREATMENT OF NEUROENDOCRINE} CARCINOMAS: IS THE SUCCESS ACHIEVED?

\section{Morgunov L.Ju.}

Moscow State University of Medicine and Dentistry named after A.I. Evdokimov

Ulitsa Delegatskaya, 20/1, Moskva, 127473, Russia

\section{Abstract:}

Diagnosis and treatment of functioning and non-functioning neuroendocrine tumors is a challenging task. Surgeru remains the only radical method of treatment of these tumors, but tumors often are diagnosed at the stage of metastasis, when only palliative treatment is possible. Octreotide in patients with neuroendocrine tumors remains a drug with proven efficacy that can suppress the clinical symptoms of carcinoid syndrome and has antiproliferative activity, which can improve the quality of life and prolong survival.

Нейроэндокринные опухоли (НЭО) - гетерогенная группа опухолей, возникающих в любых органах, где имеются эндокринные клетки. В последнее время достигнут успех в создании гистологических классификационных схем этих опухолей, современных методов визуализации и более глубоком понимании молекулярных механизмов, лежащих в основе опухолевой прогрессии. НЭО классифицируются по локализации (кишечник, поджелудочная железа, желудок, легкие, почки, яичники, тимус, предстательная, молочная, щитовидная железы), степени дифференцировки, функциональности, биологическим особенностям и злокачественному потенциалу, связанному с размером опухоли и особенностями инвазии в лимфатические или кровеносные сосуды. Эти новообразования могут не давать клинической симптоматики, но, в случае функциональности, развиваются эндокринные синдромы, представляющие угрозу здоровью и жизни пациентов.

НЭО являются относительно медленно растущими новообразованиями. Большинство НЭО Всемирная организация здравоохранения относит к гистологическим группам G1 и G2 (высокодифференцированные или умеренно дифференцированные), но некоторые из них могут быть достаточно агрессивными. Наиболее распространенными являются опухоли гастроэнтеропан- 
креатической зоны, затем - бронхолегочные нейроэндокринные образования; менее частой локализацией являются яичники, яичко и гепатобилиарный тракт. Они могут быть гормонально неактивными, либо же автономно секретировать нейропептиды, вызывающие определенную клиническую симптоматику. Среди нейроэндокринных солитарные опухоли встречаются реже, чем метастатические. До 75\% пациентов с НЭО тонкой кишки и 30-85\% - поджелудочной железы диагностируются уже на стадии метастатического поражения печени. K моменту постановки первичного диагноза злокачественных НЭО метастазы определяются у $45 \%$ больных. Пятилетняя выживаемость пациентов с наличием карциноидной опухоли составляет около 50\% [1].

Карциноидные опухоли, которые встречаются с частотой 2-3 случая на 100000 человек, секретируют биологически активные амины и нейропептиды, такие, как серотонин, простагландины, нейронспецифическую енолазу, калликреин, 5-гидрокситриптофан, синаптофизин, хромогранины, инсулин, панкреатический полипептид, нейротензин, адренокортикотропный гормон (АКТГ), меланоцитстимулирующий гормон, кальцитонин, тахикинины и т.д. Клиническая картина проявляется карциноидным синдромом, который, как правило, проявляется при метастазировании первичной опухоли в печень, и тогда характерная симптоматика проявляется в 95\% случаев [2]. Симптоматика этого синдрома представлена приливами, диареей, болями в животе, диспноэ, поражением клапанов сердца, телеангиэктазиями, бронхоспазмом, слезотечением, пеллагрой и т.д.

Задачей лечения больных с НЭО является как ликвидация самой опухоли, так и подавление симптомокомплекса, обусловленного карциноидным синдромом, который сам по себе может являться причиной смерти больного. Единственным радикальным методом лечения остается хирургическое вмешательство, а при невозможности его выполнения - торможение опухолевого роста и подавление гормональной экспрессии. Комплексное использование существующих современных методов терапии позволяет значительно продлить жизнь и улучшить ее качество у больных с НЭО.

Однако целесообразными остаются и паллиативные операции, так как частичная циторедукция определяет возможность дальнейшего лекарственного воздействия в виде химиотерапии (преимущественно препаратами платины). К сожалению, в большинстве случаев из-за поздней диагностики радикальное хирургическое вмешательство невозможно, что определяется как большими размерами опухоли и ее прорастанием в жизненно важные органы, так и наличием метастазов (преимущественно в печень). Радикальное удаление первичной опухоли при НЭО является наиболее эффективным методом лечения, но уже в 65- 70\% случаев больные со злокачественными формами НЭО на момент первичного обращения уже имеют отдаленные метастазы. Это требует включения в терапию препаратов, способствующих подавлению карциноидного синдрома, например, аналога соматостатина октреотида [3].

Аналоги соматостатина используются как эффективное средство борьбы с карциноидным синдромом в те- чение последних 25 лет. Существует убедительное доказательство того, что октреотид является действенным противоопухолевым агентом, по меньшей мере, при хорошо дифференцированных НЭО тонкого кишечника и, возможно, поджелудочной железы [4].

Карциноиды в основном представляют собой медленно растущие нейроэндокринные опухоли с низкой пролиферативной активностью. В их отношении существует широкий ассортимент терапевтических возможностей с переменной эффективностью, в том числе локальная аблативная стратегия. После проведенного такого лечения некоторым пациентам не требуется дополнительного проведения терапии в течение многих лет, в зависимости от скорости прогрессирования или рецидива. Тем не менее, большинство пациентов нуждаются в системном лечении, хотя ни одно антипролиферативное средство в настоящее время не одобрено для лечения карциноидов. При этом октреотид и, в меньшей степени, интерферональфа, остаются стандартами терапии карциноидного синдрома, одновременно обладая некоторой антипролиферативной активностью. Лечение октреотидом само по себе редко приводит к ремиссии опухолевого процесса, но может увеличить время прогрессирования опухоли. Химиотерапия имеет ограниченную ценность при карциноидах с низкими индексами пролиферации. Радионуклидная терапия пептидных рецепторов также может оказаться полезной, хотя порою используется для лечения наудачу. Октреотид же остается препаратом выбора для лечения карциноидного синдрома, обладая, к тому же, антипролиферативным действием [5].

Наличие метастатического очага в печени является лучшим прогностическим маркером выживаемости независимо от первичной локализации опухоли. Если радикальная резекция или интервенционные методы лечения печеночной опухоли не представляются возможными, или метастазы не ограничиваются локализацией в печени, единственным вариантом остается системное лечение аналогами соматостатина. Ни один из существующих вариантов терапии октреотидом не направлен непосредственно на печеночные метастазы, а действует при любой локализации метастатических очагов. К сожалению, отсутствие перспективных исследований, сравнивающих различные методы лечения в однородных когортах пациентов, не позволяет определить лучшую стратегию терапии.

Стандартное консервативное лечение проводится аналогами соматостатина (октреотидом), интерфероном $\alpha$ и химиотерапией. Октреотид не только нивелирует симптомы, связанные с функционирующими опухолями, но также тормозят их рост. Из-за сложности исследований эффективности, а также высокого потенциала побочных эффектов, широкое применение интерферона- $\alpha$ в лечении метастатических нейроэндокринных опухолей ограничено. Хорошо дифференцированные нейроэндокринные опухоли не обладают высокой чувствительностью к химиотерапии из-за низкой скорости митоза, высоких уровней антиапоптического белка Bcl-2 и повышенной экспрессии гена резистентности ко многим лекарственным средствам. Поэтому наиболее перспективным пока остается применение аналогов соматостатина [6]. 
Хотя основным видом химиотерапии высокодифференцированных карцином остаются препараты платины, октреотид не перестает играть ключевую роль в коррекции симптоматики, вызванной секрецией нейропептидов. Кроме того, октреотид доказательно продемонстрировал противоопухолевую активность при карциноидах кишечника, значительно увеличивая время прогрессирования НЭО. В настоящее время созданы новые аналоги соматостатина, в частности, октреотид-депо, который эффективно применяется как в качестве монотерапии, так и в сочетании с химиотерапией или радионуклидной терапией пептидных рецепторов [7].

Октреотид был первым аналогом соматостатина, который был разработан более 30 лет назад, и в течение этого периода были накоплены данные о его эффективности и безопасности. Комбинации октреотида с таргетной терапией могут значительно улучшить результаты лечения. Данные последних исследований комбинации октреотида и ингибитора пролиферативного сигнала эверолимуса показали обнадеживающие результаты. Изучаются комбинации октреотида с другими препаратами (например, интерфероном $\alpha$, бевацизумабом, цетуксимабом и сунитинибом). Октреотид применяется для купирования симптомов НЭО на протяжении десятилетий, а также обладает противоопухолевым эффектом, заметно продлевая выживаемость без прогрессирования заболевания [8].

Октреотид оказывает противоопухолевое действие, замедляя рост НЭО путем воздействия на рецепторы соматостатина, которые имеются как в первичном очаге, так и метастазах [9]. В последнее время обоснованный интерес представляет пролонгированная форма сандостатина - октреотид-депо. Эффективный и безопасный в применении, вводимый 1 раз в месяц, он имеет преимущества перед обычным сандостатином за счет поддержания непрерывной концентрации препарата в организме, что увеличивает как его противоопухолевое, так и симптоматическое действие.

Механизм действия октреотида основан на ингибировании повышенной секреции пептидов и серотонина, продуцируемых эндокринной системой гипофиза и желудочно-кишечного тракта. В результате снижается моторика последнего, суживаются сосуды брюшной полости; описано противовоспалительное и иммуностимулирующее действие препарата, установлено взаимодействие с факторами роста, антипролиферативный и антиангиогенный эффекты [10].

Октреотид является средством патогенетической терапии при опухолях, активно экспрессирующих рецепторы к соматостатину. Он представляет собой синтетический октапептид, являющийся производным естественного гормона соматостатина и обладающий сходными с ним фармакологическими эффектами, но значительно большей продолжительностью действия. Препарат подавляет патологически повышенную секрецию гормона роста, а также пептидов и серотонина, продуцируемых в гастроэнтеропанкреатической эндокринной системе.

Молекулярные механизмы, приводящие к успешной борьбе с болезнью или коррекции симптомов функ- ционирующих НЭО, особенно при малой концентрации рецепторов сандостатина, в значительной степени неизвестны. В одном из исследований опухолевые клетки CNDT2.5 обрабатывались октреотидом продолжительностью от 1 дня до 16 месяцев, затем был проведен анализ экспрессии генов с помощью микрочипов Affymetrix. Затем оценивался рост клеток CNDT2.5 в присутствии или отсутствии 1 мкМ октреотида в различные моменты времени. Кроме того, использовалась лазерная захватывающая микродиссекции опухолевых клеток при различных стадиях заболевания для определения экспрессии на уровне транскрипции и трансляции. Значимых изменений в уровнях экспрессии соматостатиновых рецепторов зарегистрировано не было. Однако было выявлено шесть новых генов, функционирование которых регулировалось октреотидом в опухолевых клетках: аннексин A1 (ANXA1), активирующий GTP-азу Rho белок 18 (ARHGAP18) эпителиальный мембранный белок 1 (EMP1), ген, кодирующий фактор роста и дифференцировки 15 (GDF15), трансформирующий фактор роста бета (TGFBR2) и член надсемейства (лигандов) факторов некроза опухоли 15 (TNFSF15). Таким образом, октреотид оказался потенциально способным регулировать рост и дифференцировку опухолевых нейроэндокринных клеток [11].

В течение многих десятилетий октреотид использовался для облегчения симптомов приливов, диареи, бронхоспазма, связанными с секреторной активностью НЭО, но его противоопухолевая активность оставалась предметом дискуссий. Для ее изучения у пациентов с НЭО, получающих октреотид, был проведен систематический обзор публикаций MEDLINE и других Интернетресурсов. Были проанализированы результаты 17 исследований, посвященных октреотиду, которые включали изучение его противоопухолевого действия. Частичный ответ составил от 0\% до 31\%, а показатели стабильности заболевания (отсутствие прогрессирования) варьировали между 15\% и 89\%. Октреотид был единственным аналогом соматостатина, который принял участие в фазе III рандомизированного плацебо-контролируемого клинического исследования, которое изучало его противоопухолевые эффекты. После 6 месяцев лечения стабильное течение заболевания наблюдалось у $67 \%$ пациентов, отношение рисков для времени до прогрессирования заболевания составило 0,34; 95\% доверительный интервал: 0.20-0.59; $p=0,000072$. Исследования убедительно подтвердили, что терапия октеротидом замедляет рост НЭО [12].

Пациенты с неоперабельными метастатическими нейроэндокринными опухолями испытывают проявления гормональной гиперсекреции, включающие диарею, приливы и бронхоспазм, которые существенно ухудшают качество жизни, а лечение октреотидом длительного действия облегчает эти симптомы. Хотя высокие дозы октреотида часто требуются для адекватного контроля клинической картины заболевания, их эффективность и влияние на симптоматику недостаточно описаны в литературе. Al-Efraij K. с соавт. провели ретроспективный обзор лечения неоперабельных пациентов с НЭО и метастатическим поражением, получавших дозу октреотида пролонгированного действия 30 мг и выше в период 
с января 2005 года по декабрь 2011 года. Увеличение дозы октреотида было связано с эффективным нивелированием симптомов НЭО у пациентов, которые были невосприимчивы к стандартным дозам и не получали никакой противоопухолевой терапии. Снижение уровней сывороточного хромогранина А (плазматического маркера нейроэндокринных опухолей) и 5-гидроксииндолуксусной кислоты (показателя обмена серотонина) составило, по меньшей мере, $31 \%$ и 23\% соответственно. В данном исследовании ретроспективный анализ не показал визуального сокращения опухолей в объеме. Таким образом, было продемонстрировано, что более высокие дозы октреотида улучшали клиническую симптоматику у пациентов с НЭО, не получающих химиотерапии [13].

Применение октреотида сопровождается снижением концентрации серотонина в плазме и экскреции 5-гидроксииндолуксусной кислоты с мочой. При опухолях, характеризующихся гиперпродукцией вазоактивного интестинального пептида (ВИПомах), это приводит у большинства больных к уменьшению тяжелой секреторной диареи, которая характерна для данного состояния, что в свою очередь приводит к улучшению качества жизни больного. Одновременно происходит уменьшение сопутствующих нарушений электролитного баланса, например, гипокалиемии, что позволяет отменить энтеральное и парентеральное введение жидкости и электролитов. Клиническое улучшение обычно сопровождается уменьшением (вплоть до нормальных значений) концентрации вазоактивного интестинального пептида в плазме [14].

НЭО желудочно-кишечного тракта встречаются достаточно редко и составляют менее $2 \%$ опухолей данной локализации. Их распространенность составляет 1-3 случая на 100000 населения в год. Тем не менее, она возросла в 5 раз в течение последних 30 лет, что связано с развитием современных методов визуализации новообразований. При этом ежегодное процентное увеличение составляет 5,8\%. Регрессионный анализ говорит о дальнейшем росте этой заболеваемости, которая в ближайшие 2-3 года может составить 8 случаев на 100000 населения [15]. Modlin I. М. с соавт. подтвердили, что при аутопсии карциноиды брюшной полости встречаются с частотой 8,4 на 100000 человек [16]. Консервативное лечение аналогами соматостатина позволяет воздействовать на клинические симптомы карциноидного синдрома, сопровождающего нейроэндокринные опухоли, а в 30-50\% случаев - стабилизировать их рост.

В России в настоящее отсутствуют статистические данные по заболеваемости НЭО желудочно-кишечного тракта, что затрудняет анализ результатов лечения и выработку диагностической тактики [17].

Из-за ингибирующего действия на секрецию гормонов октреотид играет ключевую роль в симптоматическом лечении гормонально-секретирующих НЭО.При этом клинические наблюдения подтвердили, что октреотид способен ингибировать рост НЭО, что убедительно продемонстрировало исследование PROMID, результаты которого были опубликованы в 2009 году. Оно также показало увеличение выживаемости и отсутствие прогрессирования опухолевой активности [18].
При лечении НЭО ЖКТ и поджелудочной железы у пациентов, которым подкожное введение октреотида обеспечивает адекватный контроль проявлений заболевания, рекомендуемая начальная доза октреотида-депо составляет 20 мг каждые 4 недели. Подкожное введение следует продолжать еще в течение 2 недель после первого введения октреотида-депо. У пациентов, не получавших ранее октреотид, рекомендуется начинать лечение именно с подкожного введения октреотида в дозе 0,1 мг 3 раза в сутки в течение относительно короткого периода времени (примерно 2 недели) с целью оценки его эффективности и общей переносимости. Только после этого назначают октреотид-депо по вышеприведенной схеме. В случае, когда терапия октреотидом-депо в течение 3 месяцев обеспечивает адекватный контроль клинических проявлений и биологических маркеров заболевания, можно снизить дозу октреотида-депо до 10 мг, назначаемую каждые 4 недели. В тех случаях, когда после 3 месяцев лечения октреотидом-депо удалось достичь лишь частичного улучшения, дозу препарата можно увеличить до 30 мг каждые 4 недели. На фоне лечения октреотидом-депо в отдельные дни возможно усиление клинических проявлений, характерных для эндокринных опухолей жКТ и поджелудочной железы. В этих случаях рекомендуется дополнительное подкожное введение октреотида в дозе, применявшейся до начала лечения октреотидом-депо. Это может происходить, главным образом, в первые 2 месяца лечения, пока не достигнуты терапевтические концентрации октреотида в плазме.

Панкреатические НЭО представлены как функционирующими опухолями, так и новообразованиями без определенной функциональной активности [19]. Нефункционирующие панкреатические НЭО секретируют панкреатический полипептид, хромогранин А, нейроспецифическую енолазу, хорионический гонадотропин, кальцитонин, нейротензин и иные пептиды. Такие панкреатические НЭО являются нефункционирующими опухолями, хотя среди НЭО немалую долю занимают и гормонально-активные новообразования [20]. НЭО гастроэнтеропанкреатической зоны являются редкими опухолями, которые имеют множество клинических особенностей. Они выделяют пептиды и нейроамины, которые вызывают различные клинические синдромы, в том числе, карциноидный. Пациентам, которые не отвечают или перестают реагировать на стандартные дозы октреотида, назначают высокие дозы этого препарата, которые приводят к снмижению или нормализации выработки биологически активных аминов и нейропептидов [2].

Целью исследования Strosberg J. R. с соавт. было изучение причин увеличения дозы октреотида для улучшения симптоматики карциноидного синдрома у пациентов с НЭО, которые получали лечение возрастающими дозами препарата в трех специализированных онкологических центрах. Изучалась медицинская документация пациентов с диагнозом карциноидного синдрома или НЭО поджелудочной железы, которые получили дозу октреотида 30 мг и более каждые 4 недели в период с 2000 по 2012 год. Среди 239 пациентов с НЭО было 53\% мужчин, средний возраст при первой эскалации дозы 
составил 60 лет (стандартное отклонение (SD) 11 лет), среднее время от начала увеличения дозы октреотида 1,7 лет (SD 2,0 года). Основными причинами, приведшими к увеличению дозы, были проявления карциноидного синдрома или иная гормональная активность (62\%), а также рентгенологически подтвержденное прогрессирование опухоли (28\%). Наиболее частыми изменением дозы было увеличение ее до 40 мг каждые 4 недели (71\%) и 60 мг каждые 4 недели (18\%). Среди 90 пациентов, у которых до первого повышения дозы отмечались приливы, 73 (81\%) либо перестали испытывать их вовсе, либо симптоматика стала невыраженной. Из 107 пациентов с диареей, 85 (79\%) отметили улучшение или ее отсутствие после первой эскалации дозы. Это исследование показывало, что увеличение дозы вводимого октреотида выше стандартной (30 мг каждые 4 недели) может привести к улучшению контроля проявлений карциноидного синдрома [21].

Jann H. c соавт. оценивали антипролиферативное действие октреотида у больных злокачественными НЭО поджелудочной железы. Ретроспективно анализировались истории болезни 43 пациентов, получавших лечение октреотидом как препаратом первой линии. Целью исследования были изучение параметров заболевания в соответствии с критериями RECIST, оценка полного или частичного исчезновения опухоли, эффект от терапии за 12 месяцев и время опухолевой прогрессии. Средний возраст пациентов (16 женщин и 27 мужчин) исходно составил 54,7 $\pm 11,86$ лет. До начала терапии 39 из 43 пациентов были классифицированы как имеющие IV стадию заболевания по классификации TNM. Опухоли также верифицировались на основе определения титра антител MIB-1, индекса пролиферации Ki67 как G1 (N = 8), G2 (N = 30) или неизвестные ( $N=5)$. Радиоиндикатор OctreoScan оказался положительным у 37 пациентов, отрицательным - у 2, и был неизвестен в 4 случаях. 19 пациентов имели функционирующие, а 24 - нефункционирующие опухоли. Медиана общей выживаемости составила 98 месяцев, а медиана выживаемости без прогрессирования составила 13 месяцев. Анализ показал статистически значимый уровень медианы выживаемости без прогрессирования опухоли для Кi67> 10\% с Кi67 <5\% (p = 0,009) и Ki67 5-10\% ( $=0,036)$. Таким образом, октреотид может рассматриваться в качестве препарата первой линии как антипролиферативный препарат у пациентов со злокачественной НЭО поджелудочной железы. Пациенты с индексом пролиферации <10\% показали более значимый результат по сравнению с имеющими более высокий индекс пролиферации [22].

Интересно описание случая о подкожном непрерывном введении октреотида пациенту с НЭО поджелудочной железы с несколькими метастазами в печени и асцитом в целях облегчения его общего состояния. Уровень опухолевого маркера нейронспецифической енолазы после лечения оказался в границах нормального диапазона, и после проведенной терапии был также подтвержден кистозный некроз опухоли. Авторы работы, основываясь на собственном опыте и данных литературы, полагают допустимым такой вариант лечения при злокачественных НЭО поджелудочной железы у пациен- тов, которым введение противоопухолевых препаратов противопоказано из-за плохого общего состояния [23].

Японские исследователи описали два случая эффективного использования октреотида у пациентов с НЭО и метастатическим поражением печени. Первый случай описан у 38-летнего мужчины, у которого была произведена операция панкреатодуоденальной резекции и гепатэктомии при НЭО поджелудочной железы с множественными метастазами в печень. Рецидивирующая опухоль была обнаружена в печени через 3 месяца после операции. Второй случай был связан с 61-летней женщиной, которая была оперирована по поводу НЭО поджелудочной железы. Множественные метастазы в печени были обнаружены 29 месяцев спустя. Оба пациента получили комбинированную терапию, включающую трансартериальную химиоэмболизацию и октреотид, которая увеличила выживаемость без прогрессирования роста опухоли в обоих случаях. Хотя описаны лишь 2 случая успешного лечения пациентов со столь тяжелой патологией, в обоих случаях мультимодальное лечение оправдало себя полностью [24].

Радикальное лечение НЭО у пожилых людей само по себе несет известные риски. Октреотид длительного действия, используемый для лечения карциноидного синдрома и диареи, может задержать опухоли рост у пациентов с НЭО. Shen C. с соавт. показали, что результаты использования октреотида у пожилых людей в реальной клинической практике и его влияния на выживаемость должным образом не документированы. Оценивая конечные результаты исследований, авторы изучили базу данных Medicare и выявили пациентов в возрасте $\geq$ 65 лет, у которых в период с июля 1999 года до декабря 2007 года был диагностирован карциноидный синдром. Показания к назначению октреотида определялись исходя из требований Medicare. Для оценки влияния октреотида на выживание использовали модель пропорциональных рисков Кокса. 2450 из 4848 пациентов с болезнью на поздней стадии (51\%) и 81 из 807 пациентов с солитарной НЭО (10\%) получали лечение октреотидом в течение 6 месяцев после постановки диагноза. Многомерный логистический регрессионный анализ показал, что пациенты с поздними стадиями заболевания, более старшего возраста ( $\geq 80$ лет против 65-69 лет) (отношение шансов [ОШ] 0,43; 95\% доверительный интервал [95\% ДИ], 0.23-0.81), женщин (ОШ, 0,62; 95\% ДИ 0.40-0.97) в недостаточной степени получали октреотид в качестве терапии. Многофакторная модель пропорциональных рисков показала, что октреотид обеспечил значительное преимущество 5-летней выживаемости для больных с отдаленными стадиями заболевания (отношение рисков, 0.61; $\mathrm{P} \leq 0,001)$. Результаты этого ретроспективного исследования предположили возможную выгоду при использовании октреотида у пациентов пожилого возраста, страдающих НЭО с отдаленными метастазами. Результаты данного исследования также показывают, что октреотид используется недостаточно, несмотря на рекомендации Medicare [25].

Хотя заболеваемость НЭО низка, их распространенность возрастает из-за медленного протекания болезни. С 1 июля 2008 года по 1 июля 2013 года Uhlyarik A. с со- 
авт. исследовали 56 пациентов с хорошо или умеренно дифференцированными нейроэндокринными опухолями; 36 пациентов с метастатическим поражением прошли курс лечения, в то время как 17 пациентов, оперированных радикально, консервативной терапии не получали. Все пациенты с метастазами получили лечение октреотидом длительного действия, причем терапия основывалась на локализации первичного очага, степени дифференцировки, индексе пролиферации Кi67 и результатах сцинтиграфии соматостатиновых рецепторов с мета-йод-бензил-гуанидином. Авторы представили подробную историю болезни пациента с НЭО кишечника, раковым синдромом и метастатическим поражением, который получал октреотид длительного действия и радионуклидную терапию пептидных рецепторов. Было достигнуто уменьшение размеров опухоли и разрешение проявлений карциноидного синдрома. Данная история болезни подтвердила предыдущие наблюдения, показывающие, что октреотид длительного действия в сочетании с радионуклидной терапией пептидных рецепторов может обеспечить длительное выживание с хорошим качеством жизни у пациентов с НЭО и метастатическим поражением, которые сопровождаются карциноидным синдромом [26].

Аналоги соматостатина представляют собой один из основных терапевтических вариантов у пациентов с функционирующими высокодифференцированными НЭО. Однако не существует никаких исследований, специально ориентированных на НЭО, которые ассоциированы с синдромом множественной эндокринной неоплазии 1 типа (МЭН1).

Ramundo V. C соавт. провели ретроспективную оценку лечения 40 пациентов с МЭН1. 20 из них с признаками одного или нескольких проявлений МЭН1, связанных с дуодено-панкреатическими НЭО размерами <20 мм (возрастной диапазон 26-61 лет), получали октреотид длительного действия в качестве первой линии терапии. Продолжительность курса лечения составила 12-75 месяцев. Уменьшение опухоли наблюдалось в 10\%, стабилизация заболевания - в 80\% и прогрессирование заболевания - в 10\% случаев. У шести пациентов с аномально повышенным уровнем хромогранина А CgA, гастрина и/или концентрации инсулина в сыворотке крови, произошло значительное клинико-гормональное улучшение (в 100\% случаев), которое оставалось стабильным в течение всего времени наблюдения. Таким образом, терапия октреотидом является безопасной и эффективной у пациентов с ранними стадиями синдрома МЭН1, что говорит о том, что раннее начало лечения октреотидом у таких пациентов является патогенетически обоснованным [27].

Yoshida Y. C соавт. сообщили о 41-летней женщине, страдающей злокачественной НЭО с множественными метастазами в печень. Результаты КТ и МРТ, а также гистологических и иммуногистохимических анализов указывали на высокую гормональную активность и распространенность процесса. Была применена комбинированная терапия S-1 и гемцитабином вкупе с октреотидом длительного действия, в результате которой отмечена стабилизация роста опухоли и хорошее качество жизни в течение 7 месяцев, а период выживания составил 15 месяцев. Хотя опухоль оказалась низкодифференцированной, терапия оказалась эффективной [28].

Аналоги соматостатина являются стандартом лечения пациентов с функционирующими НЭО гастро-энтеро-панкреатической локализации. Положительные результаты их воздействия получены в более чем 70\% наблюдений у пациентов со злокачественными функционирующими НЭО. Аналогичные результаты получены у больных с гормонсекретирующими доброкачественными опухолями поджелудочной железы. Хотя использование октреотида как антипролиферативного средства было начато лишь недавно, ретроспективные исследования показали стабилизацию опухолевого роста у более чем 50\% пациентов с прогрессирующим ростом опухоли. Результаты III фазы рандомизированного исследования PROMID показали, что медиана времени до прогрессирования у больных с карциноидными опухолями кишечника, у пациентов, получающих октреотид, была более чем в два раза выше по сравнению с пациентами, получавшими плацебо [29]. Аналогичные данные получены в отношении лечения октреотидом длительного действия НЭО поджелудочной железы [30].

Заболеваемость колоректальными НЭО растет, и такие пациенты имеют особенно неблагоприятный прогноз. Варианты лечения ограничены, а время выживания за последнее десятилетие не увеличилось. Ниже приведены результаты рандомизированного, двойного слепого, плацебо-контролируемого исследования RADIANT-2 по сопоставлению перорального введения 10 мг эверолимуса с плацебо, либо в сочетании с внутримышечным введением 30 мг октреотида каждые 28 дней. В исследовании приняли участие пациенты старше 18 лет с низкодифференцированными или среднедифференцированными неоперабельными местно-распространенными НЭО, либо имеющие отдаленные метастазы. Прогрессирование заболевания определялось с помощью радиологической оценки в течение последних 12 месяцев. 429 человек были рандомизированы; 357 участников вышли из исследования и один человек скончался. Медиана выживаемости без прогрессирования составила 16,4 (95\% ди 13,7-21,2) месяца в группе, получающей эверолимус плюс октреотид и 11,3 (95\% ДИ 8,4-14,6) месяца в группе, получающей плацебо плюс октреотид (отношение рисков 0,77, 95\% ди 0,59-1,00; $\mathrm{P}=0,026$ ). Побочные эффекты класса 1 или 2 (эверолимус плюс октреотид по сравнению с плацебо плюс эверолимус) включали стоматит (62\% против 14\%), сыпь (37\% против $12 \%)$, усталость (31\% против 23\%) и диарею (27\% против $16 \%$ ). Таким образом, комбинация эверолимуса и октреотида по сравнению с плацебо и эверолимусом улучшила выживаемость без прогрессирования заболевания у пациентов с запущенными НЭО и раковым синдромом. Лечение эверолимусом вкупе с октреотидом показало значительные преимущества и лучшие результаты для пациентов с распространенным колоректальным НЭО по сравнению с комбинацией плацебо- эверолимус [31].

Интересны данные отечественного исследования, в которое были включены 67 больных с морфологически подтвержденным диагнозом НЭО различной локализации. Все больные имели диссеминированный опухолевый про- 
цесс. У всех пациентов перед включением в исследование было зарегистрировано прогрессирование опухолевого процесса по данным УЗИ или КТ. Иммуногистохимическое исследование выполнено 53 больным, 14 больных имели только цитологическую верификацию диагноза.

Всего лечение получали 67 больных с 2004 по 2013 гг. Проводилась биотерапия: аналоги соматостатина в дозе 20 мг и в дозах 30-40 мг в монорежиме и в сочетании с интерфероном $\alpha$ в дозе 3 млн. МЕ 3 раза в неделю подкожно. Лечение проводили аналогами соматостатина пролонгированного действия в дозах от 20 до 40 мг. Октреотид-депо получали 47 пациентов, 17 из которых получали его как самостоятельный вид лечения, 30-в сочетании с химиотерапией для купирования карциноидного синдрома. Эффективность лечения оценивали по контролю роста опухоли и времени до прогрессирования. До начала лечения и каждые последующие 3 месяца проводилось контрольное обследование: КТ или УзИ брюшной полости и периферических лимфатических узлов, КТ или рентгенография грудной клетки. Оценка ответа опухоли на лечение проводилась в соответствии с критериями RECIST (Response Evaluation Criteria In Solid Tumors). Контроль симптоматики карциноидного синдрома оценивали по опросникам качества жизни, изменению частоты приливов и диареи. Клиническим ответом считалось снижение приливов до менее 1 раза в неделю и уменьшение частоты диареи менее 4-х раз в сутки или, если диарея отмечалась 2-4 раза в сутки - уменьшение ее частоты, а также исчезновение болей в животе.

Симптоматический эффект октреотида-депо был получен у всех пациентов, препарат позволил контролировать симптомы карциноидного синдрома у всех исследуемых. Препарат показал хорошую переносимость, а его применение не увеличивало токсичности иммуно-и химиотерапии [32].

Доклинические и клинические исследования показали синергическое действие между октреотидом и ингибитором протеинкиназ эверолимусом. Активность и безопасность эверолимуса оценивали в сочетании с октреотидом длительного действия у пациентов с НЭО гастроэнтеропанкреатического тракта и легких. Многоцентровое исследование, проведенное с использованием 2-ступенчатого метода минимакса Саймона, было посвящено терапии ранее не получавшим лечение пациентам с распространенными высокодифференцированными НЭО гастроэнтеропанкреатической зоны и легких. Пациенты получали эверолимус в дозе 10 мг в день в сочетании с октреотидом в дозе 30 мг каждые 28 дней. Первичной конечной точкой была частота объективного ответа (ЧОО). Всего в исследовании приняли участие 50 пациентов (средний возраст 60,5 лет). Первичная локализация опухолей была: в поджелудочной железе (14 пациентов), легких (11 пациентов), подвздошной кишке (9 человек), тощей и двенадцатиперстной кишке (у 2 больных) и неизвестной локализации (14 пациентов). У тринадцати пациентов (26\%) имел место карциноидный синдром. чОО составила 18\%; $2 \%$ пациентов имели полный ответ, $16 \%$ - частичный и 74\% достигли стабилизации заболевания. Рассчитанный клинический эффект составил 92\%. Сочетанная терапия эверолиму- сом с октреотидом оказалась эффективной, хорошо переносилась, и, как показало исследование, может использоваться в качестве первой линии терапии у пациентов с НЭО [33].

Хотя НЭО наиболее часто локализуются в желудочнокишечном тракте или легких, они могут порою верифицироваться в самых неожиданных местах. Lynggård L. A. с соавт. описали 56-летнюю женщину, страдающую от карциноидного синдром, вызванного выделением большого количества серотонина опухолью гипофиза. Она в течение многих лет ощущала эпизоды сердцебиения, одышки и приливов. У пациентки подозревали наличие кардиальной патологии, что привело к задержке правильного диагноза, пока анализы крови не выявили повышенного содержания серотонина и хромогранина А в плазме. Сцинтиграфия рецепторов соматостатина показала единственный положительный фокус в проекции гипофиза, а МРТ выявило гетерогенный очаг с интраи супраселлярным ростом в этой области. После предварительного лечения октреотидом, которое привело к улучшению клинической симптоматики, пациентке была выполнено транскраниальное удаление опухоли. Это привело к клиническому улучшению и нормализации параметров, полученных при сцинтиграфии рецепторов соматостатина, а также стабилизации уровней серотонина и хромогранина А. Это был первый случай выявления и успешного лечения серотонин-продуцирующей опухоли в гипофизе [34].

Синдром эктопической продукции АКТГ - редкое заболевание, которое часто ассоциируется с тяжелым гиперкортицизмом. Оптимальным решением является хирургическое иссечение опухоли. Авторы приводят клинический случай, когда 33-летний пациент был госпитализирован в 1993 году с клиническими проявлениям синдрома Кушинга. У него отмечались высокие уровни АКТГ в плазме и резко повышенное выделение свободного кортизола с мочой, которая не была подавлена высокими дозами дексаметазона. Гипофиз на МРТ выглядел нормально. КТ грудной клетки выявила 1,7 cм узелок в левом легком. Тонкоигольная трансторакальная биопсия, а также иммуноцитохимические и ультраструктурные исследования показали наличие АКТГ-продуцирующей нейроэндокринной карциномы с отдаленными метастазами. Исходно пациент получал химиотерапию вместе с введением октреотида с 1998 года, продемонстрировав благоприятный клинический, биохимический и визуальный ответ. Авторы подчеркнули отличный долгосрочный ответ на медикаментозную терапию с октреотидом, давшую превосходный антипролиферативный эффект [35].

Лечение НЭО представляет собой значительную проблему из-за гетерогенных клинических проявлений и разной степени агрессивности НЭО. Недавно завершенная 3-ья фаза нескольких исследований, в которых проверяли эффекты октреотида, сунитиниба и эверолимуса продемонстрировала возможность получения существенного позитивного ответа при лечении НЭО. Тем не менее, есть много аспектов лечения нейроэндокринных опухолей, которые остаются неясными и спорными. Североамериканское Общество по изучение НЭО 
в 2010 году опубликовало ряд руководящих принципов (Консенсус), в которых содержатся схемы лечения пациентов со злокачественными НЭО. В частности, использование аналогов соматостатина рекомендовано при наличии карциноидного синдрома, а доступными препаратами продолжают оставаться октреотид и ланреотид. В Консенсусе подчеркивается, что с течением времени пациенты с карциноидным синдромом могут стать резистентными к аналогам соматостатина, поэтому необходимо увеличение дозы вводимого препарата [36].

\section{Список литературы:}

1. Kaltsas G. A., Besser G.M., Grossman A.B. The Diagnosis and medical management of advanced neuroendocrine tumors. Endocr Rev. 2004; 25 (3): 458-511.

2. Baldelli $R$, Barnabei A, Rizza L, Isidori AM, Rota $F, D i$ Giacinto P, Paoloni A, Torino F, Corsello SM, Lenzi A, Appetecchia M. Somatostatin analogs therapy in gastroenteropancreatic neuroendocrine tumors: current aspects and new perspectives. Front Endocrinol (Lausanne). 2014; 5: 7 .

3. Chuang CC, Bhurke S, Chen SY, Brulais S, Gabriel S. Clinical characteristics, treatment patterns, and economic burden in patients treated for neuroendocrine tumors in the United States: a retrospective cohort study. J Med Econ. 2015; 18 (2): 126-136.

4. Toth M. Drug therapy for neuroendocrine tumours. Orv Hetil. 2013;154 (39): 1556-1564.

5. Pavel M, Kidd M, Modlin I. Systemic therapeutic options for carcinoid. Semin Oncol. 2013; 40 (1): 84-99.

6. Demirkan BH, Eriksson B. Systemic treatment of neuroendocrine tumors with hepatic metastases. Turk J Gastroenterol. 2012; 23 (5): 427-437.

7. Bergsland EK. The evolving landscape of neuroendocrine tumors. Semin Oncol. 2013; 40 (1): 4-22.

8. Wolin EM. The expanding role of somatostatin analogs in the management of neuroendocrine tumors. Gastrointest Cancer Res. 2012; 5 (5): 161-168.

9. Young $\mathrm{K}$, lyer R, Morganstein D, Chau I, Cunningham D, Starling N. Pancreatic neuroendocrine tumors: a review. Future Oncol. 2015; 11 (5): 853-864.

10. Jia W.D., Xu G.L., Sun H.C., et al. Effect of octreotide on angiogenesis induced by hepatocellular carcinoma in vivo//Hepatobiliary Pancreat Dis Int. 2003; 2 (3): 404-409.

11. Li SC, Martijn C, Cui T, Essaghir A, Luque RM, Demoulin JB, Castano JP, Oberg K, Giandomenico V. The somatostatin analogue octreotide inhibits growth of small intestine neuroendocrine tumour cells. PLoS One. 2012; 7 (10): e48411.

12. Sideris $L$, Dube $P$, Rinke A. Antitumor effects of somatostatin analogs in neuroendocrine tumors. Oncologist. 2012; 17 (6): 747-755.

13. Al-Efraij K, Aljama MA, Kennecke HF. Association of dose escalation of octreotide long-acting release on clinical symptoms and tumor markers and response among patients with neuroendocrine tumors. Cancer Med. 2015. doi: 10.1002/cam4.435.

14. Adam N, Lim SS, Ananda V, Chan SP. VIPoma syndrome: challenges in management. Singapore Med J. 2010; 51 (7): e129-132.

15. Нейроэндокринные опухоли: руководство для врачей. Под ред.М. Кэплина, Л. Квопса. М., Практическая медицина, 2010. 224 c.

16. Modlin I.M., Lye K.D., Kidd M. A 5-decade analysis of 13,715 carcinoid tumours. Cancer. 2003; 97: 934-959.

17. Нейроэндокринные опухоли желудочно-кишечного тракта.
Таким образом, октреотид, и, в частности, его пролонгированная форма - октреотид-депо, с успехом применяется как в качестве монотерапии, так и комбинированного лечения пациентов с НЭО любой локализации. Препарат рекомендован для купирования проявлений карциноидного синдрома и обладает при этом антипролиферативной активностью. Терапия октреотидом-депо эффективна у пациентов любого возраста, препарат безопасен в применении и имеет широкие перспективы для дальнейшего использования.

Принципы диагностики и лечения/Под ред. В. А. Горбунова, А. В. Егорова, А. В. Кочатков. - М., 2009. - 196 с.

18. Igaz P. Efficacy of somatostatin analogues in the treatment of neuroendocrine tumours based on the results of recent clinical trials. Orv Hetil. 2014; 155 (48): 1908-1912.

19. Oberg K. Pancreatic endocrine tumors. Semin. Oncol. 2010; 37: 594-618

20. Kaltsas G., Androulakis I. I., de Herder W. W. et al. Paraneoplastic syndromes secondary to neuroendocrine tumours. Endocr. Relat. Cancer. 2010; 17: 173-193.

21. Strosberg JR, Benson AB, Huynh L, Duh MS, Goldman J, Sahai V, Rademaker AW, Kulke MH. Clinical benefits of above-standard dose of octreotide LAR in patients with neuroendocrine tumors for control of carcinoid syndrome symptoms: a multicenter retrospective chart review study. Oncologist. 2014; 19 (9): 930-936.

22. Jann H, Denecke T, Koch M, Pape UF, Wiedenmann B, Pavel M. Impact of octreotide long-acting release on tumour growth control as a first-line treatment in neuroendocrine tumours of pancreatic origin. Neuroendocrinology. 2013; 98 (2):137-143.

23. Miura $M$, Shibahara $H$, Morita $K$, Matsui $K$, Itoh $Y$, Shimizu J, Takeuchi A, Narita M, Nishimura D, Katada N. Subcutaneous continuous injection of octreotide decreased the tumor marker levels and induced cystic necrosis of the tumors in a case of pancreatic neuroendocrine tumor with multiple hepatic metastases. Nihon Shokakibyo Gakkai Zasshi. 2015; 112 (3): 537-546.

24. Goto W, Shimizu S, Kotsuka M, Sakae M, Kanazawa A, Tsukamoto $T$, Yamashita $Y$, Nishiguchi $Y$ Two cases of neuroendocrine tumors of the pancreas with multiple liver metastases treated by use of degradable starch microspheres and octreotide. Gan To Kagaku Ryoho. 2014; 41 (12): 2139-2141

25. Shen C, Shih YC, Xu Y, Yao JC. Octreotide long-acting repeatable use among elderly patients with carcinoid syndrome and survival outcomes: a population-based analysis. Cancer. 2014; 120 (13): 2039-2049.

26. Uhlyarik A, Lahm E, Vachaja J, Papai Z. Treatment of metastatic midgut neuroendocrine tumour associated with carcinoid syndrome. Orv Hetil. 2014; 155 (5): 194-198.

27. Ramundo V, Del Prete M, Marotta V, Marciello F, Camera L, Napolitano V, De Luca L, Circelli L, Colantuoni V, Di Sarno A, Carratu AC, de Luca di Roseto C, Colao A, Faggiano A; Multidisciplinary Group for Neuroendocrine Tumors of Naples. Impact of long-acting octreotide in patients with early-stage MEN1-related duodeno-pancreatic neuroendocrine tumours. Clin Endocrinol (Oxf). 2014; 80 (6): 850-855.

28. Yoshida Y, Sugawara N, Minami T, Iwata N, Ikeda K, Endoh T, Sasano $\mathrm{H}$. A case of pancreatic neuroendocrine tumor with excessively-advanced liver metastasis treated with S-1/GEM combination chemotherapy plus the long-acting somatostatin analogue octreotide. Nihon Shokakibyo Gakkai Zasshi. 2013; 110 (4): 660-668.

29. Toumpanakis C, Caplin ME. Update on the role of somatostatin analogs for the treatment of patients with 
gastroenteropancreatic neuroendocrine tumors. Semin Oncol. 2013; 40 (1):56-68. doi: 10.1053/j.seminoncol.2012.11.006

30. Igarashi $H$, Hijioka $M$, Lee L, Ito T. Biotherapy of pancreatic neuroendocrine tumors using somatostatin analogs. J Hepatobiliary Pancreat Sci. 2015. doi: 10.1002/jhbp. 227.

31. Castellano D, Bajetta E, Panneerselvam A, Saletan S, Kocha W, O'Dorisio T, Anthony LB, Hobday T; RADIANT-2 Study Group. Everolimus Plus Octreotide Long-Acting Repeatable in Patients With Colorectal Neuroendocrine Tumors: A Subgroup Analysis of the Phase III RADIANT-2 Study. Oncologist. 2013; 18 (1): 46-53.

32. Емельянова Г.С., Маркович А.А, Кузьминов А. Е., Орел Н.Ф., Горбунова В.А. «Применение отечественного аналога пролонгированного соматостатина Октреотид-депо ${ }^{\mathrm{TM}}$ в дозах 20-40 мг у больных НЭО. Опыт отделения химиотерапии РОНЦ» Онкология, гематология и радиология, 2012, N 4, стр. 3-6.

33. Bajetta E, Catena L, Fazio N, Pusceddu S, Biondani P, Blanco G, Ricci S, Aieta M, Pucci F, Valente M, Bianco N, Mauri CM,

\section{References:}

1. Kaltsas G.A., Besser G. M., Grossman A.B. The Diagnosis and medical management of advanced neuroendocrine tumors. Endocr Rev. 2004; 25 (3): 458-511.

2. Baldelli R, Barnabei A, Rizza L, Isidori AM, Rota F, Di Giacinto P, Paoloni A, Torino F, Corsello SM, Lenzi A, Appetecchia M. Somatostatin analogs therapy in gastroenteropancreatic neuroendocrine tumors: current aspects and new perspectives. Front Endocrinol (Lausanne). 2014; 5 : 7.

3. Chuang CC, Bhurke S, Chen SY, Brulais S, Gabriel S. Clinical characteristics, treatment patterns, and economic burden in patients treated for neuroendocrine tumors in the United States: a retrospective cohort study. J Med Econ. 2015; 18 (2): 126-136.

4. Toth M. Drug therapy for neuroendocrine tumours. Orv Hetil. 2013;154 (39): 1556-1564.

5. Pavel M, Kidd M, Modlin I. Systemic therapeutic options for carcinoid. Semin Oncol. 2013; 40 (1): 84-99.

6. Demirkan BH, Eriksson B. Systemic treatment of neuroendocrine tumors with hepatic metastases. Turk J Gastroenterol. 2012; 23 (5): 427-437.

7. Bergsland EK. The evolving landscape of neuroendocrine tumors. Semin Oncol. 2013; 40 (1): 4-22.

8. Wolin EM. The expanding role of somatostatin analogs in the management of neuroendocrine tumors. Gastrointest Cancer Res. 2012; 5 (5): 161-168.

9. Young $K$, lyer $R$, Morganstein D, Chau I, Cunningham D, Starling N. Pancreatic neuroendocrine tumors: a review. Future Oncol. 2015; 11 (5): 853-864.

10. Jia W.D., Xu G.L., Sun H.C., et al. Effect of octreotide on angiogenesis induced by hepatocellular carcinoma in $\mathrm{vi}$ vo//Hepatobiliary Pancreat Dis Int. 2003; 2 (3): 404-409.

11. Li SC, Martijn C, Cui T, Essaghir A, Luque RM, Demoulin JB, Castano JP, Oberg K, Giandomenico V. The somatostatin analogue octreotide inhibits growth of small intestine neuroendocrine tumour cells. PLoS One. 2012; 7 (10): e48411.

12. Sideris $L$, Dube $P$, Rinke A. Antitumor effects of somatostatin analogs in neuroendocrine tumors. Oncologist. 2012; 17 (6): 747-755.

13. Al-Efraij K, Aljama MA, Kennecke HF. Association of dose escalation of octreotide long-acting release on clinical symptoms and tumor markers and response among patients with neuroendocrine tumors. Cancer Med. 2015. doi: 10.1002/cam4.435

14. Adam N, Lim SS, Ananda V, Chan SP. VIPoma syndrome: challenges in management. Singapore Med J. 2010; 51 (7): e129-132.
Spada F. Everolimus in combination with octreotide longacting repeatable in a first-line setting for patients with neuroendocrine tumors: an ITMO group study. Cancer. 2014; 120 (16): 2457-2463.

34. Lynggard LA, Nielsen EH, Laurberg P. Carcinoid syndrome caused by a serotonin-secreting pituitary tumour. Eur J Endocrinol. 2014; 170 (2): K5-9.

35. Rodrigues $P$, Castedo JL, Damasceno M, Carvalho D. Ectopic Cushing's syndrome caused by a pulmonary ACTH-secreting tumor in a patient treated with octreotide. Arq Bras Endocrinol Metabol. 2012; 56 (7): 461-464.

36. Kunz PL, Reidy-Lagunes D, Anthony LB, Bertino EM, Brendtro $\mathrm{K}$, Chan JA, Chen $\mathrm{H}$, Jensen RT, Kim MK, Klimstra DS, Kulke MH, Liu EH, Metz DC, Phan AT, Sippel RS, Strosberg JR, Yao JC; North American Neuroendocrine Tumor Society. Consensus guidelines for the management and treatment of neuroendocrine tumors. Pancreas. 2013; 42 (4): 557-577.

15. Neiroendokrinnye opukholi: rukovodstvo dlya vrachei. Pod red. M. Keplina, L. Kvopsa. M., Prakticheskaya meditsina, 2010. $224 \mathrm{~s}$.

16. Modlin I.M., Lye K.D., Kidd M. A 5-decade analysis of 13,715 carcinoid tumours. Cancer. 2003; 97: 934-959.

17. Neiroendokrinnye opukholi zheludochno-kishechnogo trakta. Printsipy diagnostiki i lecheniya/Pod red. V.A. Gorbunova, A. V. Egorova, A. V. Kochatkov. - M., 2009. - 196 s. (Russian)

18. Igaz P. Efficacy of somatostatin analogues in the treatment of neuroendocrine tumours based on the results of recent clinical trials. Orv Hetil. 2014; 155 (48): 1908-1912.

19. Oberg K. Pancreatic endocrine tumors. Semin. Oncol. 2010; 37: 594-618

20. Kaltsas G., Androulakis I. I., de Herder W. W. et al. Paraneoplastic syndromes secondary to neuroendocrine tumours. Endocr. Relat. Cancer. 2010; 17: 173-193.

21. Strosberg JR, Benson AB, Huynh L, Duh MS, Goldman J, Sahai V, Rademaker AW, Kulke MH. Clinical benefits of above-standard dose of octreotide LAR in patients with neuroendocrine tumors for control of carcinoid syndrome symptoms: a multicenter retrospective chart review study. Oncologist. 2014; 19 (9): 930-936.

22. Jann H, Denecke T, Koch M, Pape UF, Wiedenmann B, Pavel M. Impact of octreotide long-acting release on tumour growth control as a first-line treatment in neuroendocrine tumours of pancreatic origin. Neuroendocrinology. 2013; 98 (2):137-143.

23. Miura $M$, Shibahara $H$, Morita $K$, Matsui $K$, Itoh $Y$, Shimizu J, Takeuchi A, Narita M, Nishimura D, Katada N. Subcutaneous continuous injection of octreotide decreased the tumor marker levels and induced cystic necrosis of the tumors in a case of pancreatic neuroendocrine tumor with multiple hepatic metastases. Nihon Shokakibyo Gakkai Zasshi. 2015; 112 (3): 537-546.

24. Goto W, Shimizu S, Kotsuka M, Sakae M, Kanazawa A, Tsukamoto $T$, Yamashita $Y$, Nishiguchi $Y$ Two cases of neuroendocrine tumors of the pancreas with multiple liver metastases treated by use of degradable starch microspheres and octreotide. Gan To Kagaku Ryoho. 2014; 41 (12): 2139-2141

25. Shen C, Shih YC, Xu Y, Yao JC. Octreotide long-acting repeatable use among elderly patients with carcinoid syndrome and survival outcomes: a population-based analysis. Cancer. 2014; 120 (13): 2039-2049.

26. Uhlyarik A, Lahm E, Vachaja J, Papai Z. Treatment of metastatic midgut neuroendocrine tumour associated with carcinoid syndrome. Orv Hetil. 2014; 155 (5): 194-198.

27. Ramundo V, Del Prete M, Marotta V, Marciello F, Camera L, Napolitano V, De Luca L, Circelli L, Colantuoni V, Di Sarno 
A, Carratu AC, de Luca di Roseto C, Colao A, Faggiano A; Multidisciplinary Group for Neuroendocrine Tumors of Naples. Impact of long-acting octreotide in patients with early-stage MEN1-related duodeno-pancreatic neuroendocrine tumours. Clin Endocrinol (Oxf). 2014; 80 (6): 850-855

28. Yoshida Y, Sugawara N, Minami T, Iwata N, Ikeda K, Endoh T, Sasano H. A case of pancreatic neuroendocrine tumor with excessivelyadvanced liver metastasis treated with S-1/GEM combination chemotherapy plus the long-acting somatostatin analogue octreotide. Nihon Shokakibyo Gakkai Zasshi. 2013; 110 (4): 660-668.

29. Toumpanakis C, Caplin ME. Update on the role of somatostatin analogs for the treatment of patients with gastroenteropancreatic neuroendocrine tumors. Semin Oncol. 2013; 40 (1):56-68. doi: 10.1053/j.seminoncol.2012.11.006

30. garashi $H$, Hijioka $M$, Lee $L$, Ito T. Biotherapy of pancreatic neuroendocrine tumors using somatostatin analogs. J Hepatobiliary Pancreat Sci. 2015. doi: 10.1002/jhbp.227.

31. Castellano D, Bajetta E, Panneerselvam A, Saletan S, Kocha W, O'Dorisio T, Anthony LB, Hobday T; RADIANT-2 Study Group. Everolimus Plus Octreotide Long-Acting Repeatable in Patients With Colorectal Neuroendocrine Tumors: A Subgroup Analysis of the Phase III RADIANT-2 Study. Oncologist. 2013; 18 (1): 46-53.

32. Emel'yanova G.S., Markovich A.A, Kuz'minov A.E., Orel N.F., Gorbunova V.A. «Primenenie otechestvennogo analoga

\section{Информация об авторах:}

1. Моргунов Леонид Юльевич, д.м.н., профессор кафедры терапии, клинической фармакологии и скорой медицинской помощи ГБОУ ВПО МГМСУ имени А.И.Евдокимова Минздрава РФ prolongirovannogo somatostatina Oktreotid-depo ${ }^{\mathrm{TM}} \mathrm{v}$ dozakh 20-40 mg u bol'nykh NEO. Opyt otdeleniya khimioterapii RONTs» Onkologiya, gematologiya i radiologiya, 2012, N 4, str. 3-6. (Russian)

33. Bajetta E, Catena L, Fazio N, Pusceddu S, Biondani P, Blanco G, Ricci S, Aieta M, Pucci F, Valente M, Bianco N, Mauri CM, Spada F. Everolimus in combination with octreotide longacting repeatable in a first-line setting for patients with neuroendocrine tumors: an ITMO group study. Cancer. 2014; 120 (16): 2457-2463.

34. Lynggard LA, Nielsen EH, Laurberg P. Carcinoid syndrome caused by a serotonin-secreting pituitary tumour. Eur J Endocrinol. 2014; 170 (2): K5-9.

35. Rodrigues P, Castedo JL, Damasceno M, Carvalho D. Ectopic Cushing's syndrome caused by a pulmonary ACTH-secreting tumor in a patient treated with octreotide. Arq Bras Endocrinol Metabol. 2012; 56 (7): 461-464.

36. Kunz PL, Reidy-Lagunes D, Anthony LB, Bertino EM, Brendtro $\mathrm{K}$, Chan JA, Chen $\mathrm{H}$, Jensen RT, Kim MK, Klimstra DS, Kulke MH, Liu EH, Metz DC, Phan AT, Sippel RS, Strosberg JR, Yao JC; North American Neuroendocrine Tumor Society. Consensus guidelines for the management and treatment of neuroendocrine tumors. Pancreas. 2013; 42 (4): 557-577.

Information about authors:

1. Morgunov Leonid Yulevich, PhD, MD, professor of therapy, clinical pharmacology and medical emergencies Moscow State University of Medicine and Dentistry named after A.I. Evdokimov

\section{Оформление ссылки для цитирования статьи:}

Моргунов Л.Ю. Октреотид в лечении нейроэндокринных опухолей: достигнут ли успех? Исследования и практика в медицине. 2015; 2(2): 63-72. DOI: 10.17709/2409-2231-2015-2-2-63-72

Morgunov L. Ju. Octreotide in the treatment of neuroendocrine carcinomas: Is the success achieved? Issled. prakt. med. $2015 ; 2$ (2): 63-72. DOI: 10.17709/2409-2231-2015-2-2-63-72 